\title{
Laboratory-confirmed pandemic H1N1 influenca in hospitalized adults - findings from the Canadian Nosocomial Infection Surveillance Program (CNISP), 2009-10
}

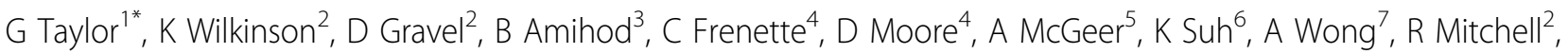 \\ Canadian Nosocomial Infection Surveillance Program
}

From International Conference on Prevention \& Infection Control (ICPIC 2011)

Geneva, Switzerland. 29 June - 2 July 2011

\section{Introduction / objectives}

To describe laboratory-confirmed pandemic H1N1 (pH1N1) influenza in adult inpatients at participating Canadian hospitals between June 2009 and May 2010 and compare to previous years' seasonal surveillance.

\section{Methods}

Adult inpatients ( $>=16$ years) with lab confirmed influenza were enrolled. Variables collected included ICU admissions and death attributed to influenza assessed 30 days after initial diagnosis.

\section{Results}

Results

Thirty-seven hospitals submitted data on 701 cases. The median age of was 49 years (range 16 - 94). Vaccine history was available for 314 cases, and $21 \%(n=65)$ reported receiving vaccine. Oseltamivir was given to $90 \%$ of the cases a median of 3 days after symptom onset (range 0 - 24). Influenza-associated admission to ICU was required for $28 \%(n=197)$. The 30 day all-cause mortality was $7 \%$; influenza was the primary cause of 20 deaths and contributed to death in a further 22 cases for an influenza-attributed mortality of $6 \%$. The mean age at death was 50 years (SD 13.8).

\section{Conclusion}

The ICU admission rate and influenza-attributed mortality were similar to three preceding influenza years, but mean age at death was significantly younger $(p<0.01)$. Antivirals were prescribed for more patients with influenza (90\%) than in previous seasons (35-47\%). The $\mathrm{pH} 1 \mathrm{~N} 1$ virus appeared outside the traditional influenza season and impacted a different age group than seasonal viruses circulating in previous years, highlighting the importance of ongoing influenza surveillance.

\section{Disclosure of interest}

None declared.

\begin{abstract}
Author details
${ }^{1}$ Univesity of Alberta, Edmonton, Canada. ${ }^{2}$ Public Health Agency of Canada, Ottawa, Canada. ${ }^{3}$ Jewish General Hospital, Canada. ${ }^{4}$ McGill University, Montreal, Canada. ${ }^{5}$ University of Toronto, Toronto, Canada. ${ }^{6}$ University of Ottawa, Ottawa, Canada. ${ }^{7}$ University of Saskatchewan, Saskatoon, Canada.
\end{abstract}

Published: 29 June 2011

doi:10.1186/1753-6561-5-S6-P81

Cite this article as: Taylor et al:: Laboratory-confirmed pandemic H1N1 influenca in hospitalized adults - findings from the Canadian Nosocomial Infection Surveillance Program (CNISP), 2009-10. BMC Proceedings 2011 5(Suppl 6):P81.

${ }^{1}$ Univesity of Alberta, Edmonton, Canada

Full list of author information is available at the end of the article

(c) 2011 Taylor et al; licensee BioMed Central Ltd. This is an open access article distributed under the terms of the Creative Commons 